\title{
The Hodge Structure of the Coloring Complex of a Hypergraph (Extended Abstract)
}

\author{
Sarah C Rundell ${ }^{1}$ and Jane H Long ${ }^{2}$ \\ ${ }^{1}$ Department of Math and Computer Science, Denison University, Granville, OH, 43023, USA \\ ${ }^{2}$ Department of Mathematics, Stephen F. Austin State University, Nacodogches, TX, 75962
}

\begin{abstract}
Let $G$ be a simple graph with $n$ vertices. The coloring complex $\Delta(G)$ was defined by Steingrímsson, and the homology of $\Delta(G)$ was shown to be nonzero only in dimension $n-3$ by Jonsson. Hanlon recently showed that the Eulerian idempotents provide a decomposition of the homology group $H_{n-3}(\Delta(G))$ where the dimension of the $j^{\text {th }}$ component in the decomposition, $H_{n-3}^{(j)}(\Delta(G))$, equals the absolute value of the coefficient of $\lambda^{j}$ in the chromatic polynomial of $G, \chi_{G}(\lambda)$. Let $H$ be a hypergraph with $n$ vertices. In this paper, we define the coloring complex of a hypergraph, $\Delta(H)$, and show that the coefficient of $\lambda^{j}$ in $\chi_{H}(\lambda)$ gives the Euler Characteristic of the $j^{t h}$ Hodge subcomplex of the Hodge decomposition of $\Delta(H)$. We also examine conditions on a hypergraph, $H$, for which its Hodge subcomplexes are Cohen-Macaulay, and thus where the absolute value of the coefficient of $\lambda^{j}$ in $\chi_{H}(\lambda)$ equals the dimension of the $j^{t h}$ Hodge piece of the Hodge decomposition of $\Delta(H)$.

Résumé. Soit $G$ un graphe simple à $n$ sommets. Le complexe de coloriage $\Delta(G)$ a été défini par Steingrímsson et Jonsson a prouvé que l'homologie de $\Delta(G)$ est non nulle seulement en dimension $n-3$. Hanlon a récemment prouvé que les idempotents eulériens fournissent une décomposition du groupe d'homologie $H_{n-3}(\Delta(G))$ où la dimension de la $j^{e}$ composante dans la décomposition de $H_{n-3}(j)(\Delta(G))$ est égale à la valeur absolue du coefficient de $\lambda^{j}$ dans le polynôme chromatique de $G, \chi_{G}(\lambda)$. Soit $H$ un hypergraphe à $n$ sommets. Dans ce texte, nous définissons le complexe de coloration d'un hypergraphe $\Delta(H)$ et nous prouvons que le coefficient de $\lambda^{j}$ dans $\chi_{H}(\lambda)$ donne la caractéristique d'Euler du $j^{e}$ sous-complexe de Hodge dans la décomposition de Hodge de $\Delta(H)$. Nous examinons également des conditions sur un hypergraphe $H$ pour lesquelles les sous-complexes de Hodge sont Cohen-Macaulay. Ainsi la valeur absolue du coefficient de $\lambda^{j}$ de $\chi_{H}(\lambda)$ est égale à la dimension du $j^{e}$ sous-complexe de Hodge dans la décomposition de Hodge de $\Delta(H)$.
\end{abstract}

Keywords: coloring complex, hypergraph, chromatic polynomial

\section{Preliminaries}

Definition 1.1 $A$ hypergraph, $H$, is an ordered pair, $(V, E)$, where $V$ is a set of vertices and $E$ is a set of subsets of the vertices of $V$. A hypergraph is said to be uniform of rank $r$ if all of its hyperedges have size $r$.

Throughout this paper, $H$ will denote a hypergraph whose vertex set $V$ is $\{1, \ldots, n\}$.

Definition 1.2 The chromatic polynomial of $H$, denoted $\chi_{H}(\lambda)$, is the number of ways to color the vertices of the hyperedges of $H$ with at most $\lambda$ colors, so that the vertices of each edge are colored with at least two colors. 
Example 1.3 Let $H$ be the hypergraph on 5 vertices with hyperedges $\{1,2,3\}$ and $\{3,4,5\}$. It follows that $\chi_{H}(\lambda)=\lambda^{5}-\left(\lambda^{3}+\lambda^{3}\right)+\lambda=\lambda^{5}-2 \lambda^{3}+\lambda$.

The following theorem is a generalization of a well-known result for the chromatic polynomial of a graph and will be used in the proof of Theorem 4.1 The same counting argument that is used to prove the deletion-contraction formula for the chromatic polynomial of a graph can be used to prove the following theorem.

Theorem 1.4 Deletion-Contraction Property [11] Let e be a hyperedge of $H$. Let $H-e$ denote the hypergraph obtained from $H$ by deleting the edge e, and let $H / e$ denote the hypergraph obtained from $H$ by identifying the vertices in $e$. Then

$$
\chi_{H}(\lambda)=\chi_{H-e}(\lambda)-\chi_{H / e}(\lambda) .
$$

Example 1.5 Let $H$ be as in Example 1.3, and let $e=\{1,2,3\}$. Then $H-e$ is the hypergraph on 5 vertices with hyperedge $\{3,4,5\}$, and $H / e$ is the hypergraph on 3 vertices with edge $\{123,4,5\}$. Then $\chi_{H-e}(\lambda)=\lambda^{2}\left(\lambda^{3}-\lambda\right)$ and $\chi_{H / e}=\lambda^{3}-\lambda$. Thus, by Theorem 1.4

$$
\chi_{H}(\lambda)=\lambda^{5}-2 \lambda^{3}+\lambda \text {. }
$$

\section{The Coloring Complex}

We begin by defining Steingrímsson's [10] coloring complex following the presentation in Jonsson [8].

Let $\left(B_{1}, \ldots, B_{r+2}\right)$ be an ordered partition of $\{1, \ldots, n\}$ where at least one of the $B_{i}$ contains a hyperedge of $H$, and let $\Delta_{r}$ be the set of ordered partitions $\left(B_{1}, \ldots, B_{r+2}\right)$.

Definition 2.1 The coloring complex of $H$ is the sequence:

$$
\ldots \rightarrow C_{r} \stackrel{\delta_{r}}{\rightarrow} C_{r-1} \stackrel{\delta_{r-1}}{\rightarrow} \ldots \stackrel{\delta_{1}}{\rightarrow} C_{0} \stackrel{\delta_{0}}{\rightarrow} C_{-1} \stackrel{\delta_{-1}}{\rightarrow} 0
$$

where $C_{r}$ is the vector space over a field of characteristic zero with basis $\Delta_{r}$ and

$$
\partial_{r}\left(\left(B_{1}, \ldots, B_{r+2}\right)\right):=\sum_{i=1}^{r+1}(-1)^{i}\left(B_{1}, \ldots, B_{i} \bigcup B_{i+1}, \ldots, B_{r+2}\right) .
$$

Notice that $\partial_{r-1} \circ \partial_{r}=0$. Then:

Definition 2.2 The $r^{\text {th }}$ homology group of $\Delta(H), H_{r}(\Delta(H)):=k e r\left(\partial_{r}\right) / i m\left(\partial_{r+1}\right)$.

It is worth noting that Hultman [7] defined a complex that includes both Steingrímsson's coloring complex and the coloring complex of a hypergraph as a special case.

For the proof of our main result, Theorem [4.1, we will need the following Lemma. Lemma 2.3 is the hypergraph version of Lemma 1.3 in Jonsson [8]. We follow the notation in Hanlon [3].

Lemma 2.3 Let $E$ be the hypergraph with the single hyperedge $e$ and the same vertices as $H$, and let $C_{r}(\Delta(H), \Delta(E))$ be the vector space spanned by ordered partitions in $\Delta_{r}(H)$ with no block containing e. Then

$$
C_{r}(\Delta(H), \Delta(E)) \cong C_{r}(\Delta(H-e)) /\left(C_{r}(\Delta(H-e)) \cap C_{r}(\Delta(E))\right)
$$

and

$$
C_{r}(\Delta(H-e)) \cap C_{r}(\Delta(E)) \cong C_{r}(\Delta(H / e))
$$


Note that $C_{*}(\Delta(H)) / C_{*}(\Delta(E))$ is the same as $C_{*}(\Delta(H), \Delta(E))$ in the relative homology sense, and we will use this fact later in some of our proofs.

\section{Eulerian Idempotents}

Recently, Hanlon [3] showed that there is a Hodge decomposition of $H_{n-3}(\Delta(G))$ for a graph $G$; we will discuss this result and its generalization to the hypergraph case. In order to describe this decomposition, we must first define and describe the Eulerian idempotents. The Eulerian idempotents have many interesting properties and have proved useful in many different algebraic and combinatorial problems. For more information on Eulerian idempotents see [2], [9], [4], and [5].

Define a descent of a permutation $\pi \in S_{n}$ to be a couple of consecutive numbers $(i, i+1)$ such that $\pi(i)>\pi(i+1)$. It follows from Loday's [9] definition that the Eulerian idempotents $e_{r}^{(j)}$ can be defined by the identity:

Definition 3.1 The Eulerian idempotents are defined by

$$
\sum_{j=1}^{n} t^{j} e_{n}^{(j)}=\sum_{\pi \in S_{n}}\left(\begin{array}{c}
n+t-\operatorname{des}(\pi)-1 \\
n
\end{array}\right) \operatorname{sgn}(\pi) \pi,
$$

where des $(\pi)$ is the number of descents of $\pi$.

There are several important properties of the Eulerian idempotents which are due to Gerstenhaber and Schack [2]. In their paper, they show that the Eulerian idempotents are mutually orthogonal idempotents and that their sum is the unit element in $\mathbb{C}\left[S_{n}\right]$. So then for any $S_{n}$-module, $M$, we have that

$$
M=\bigoplus_{j} e_{n}^{(j)} M .
$$

Notice that we can define an action of $S_{r+2}$ on $\Delta_{r}$. Namely, if $\sigma \in S_{r+2}$, then $\sigma \cdot\left(B_{1}, \ldots, B_{r+2}\right)=$ $\left(B_{\sigma^{-1}(1)}, \ldots, B_{\sigma^{-1}(r+2)}\right)$. This action then makes $C_{r}$ into an $S_{r+2}$-module.

Hanlon [3] notes (or this result can be derived from the work of Gerstenhaber and Schack [2]) in Lemma 2.1 of his paper that for any graph $G$ and for each $r, j$,

$$
\partial_{r} \circ e_{r+2}^{(j)}=e_{r+1}^{(j)} \circ \partial_{r} .
$$

This implies then that, for each $j, C_{r}^{(j)}=e_{r+2}^{(j)} C_{r}$ is a subcomplex of $\left(C_{*}(\Delta(G)), \partial_{*}\right)$. We may then consider the homology of the subcomplex, and it will be denoted by $H_{*}^{(j)}(\Delta(G))$. So then we have

$$
H_{r}(\Delta(G))=\bigoplus_{j} H_{r}^{(j)}(\Delta(G)) .
$$

The above decomposition is called the Hodge decomposition of $H_{*}(\Delta(G))$.

Hanlon [3] showed that there is a Hodge decomposition of the top homology group of $\Delta(G)$, i.e. $H_{n-3}(\Delta(G))=\bigoplus_{j=1}^{n-1} H_{n-3}^{(j)}(\Delta(G))$. Further, he showed that the dimension of the $j^{t h}$ Hodge piece is equal to the absolute value of the coefficient of $\lambda^{j}$ in the chromatic polynomial of $G$. 
In the case where $H$ is a hypergraph, the Hodge decomposition of $\Delta(H)$ can be defined by following the same process as above. However, in general, the homology of $\Delta(H)$ is not concentrated in one dimension, and thus Hanlon's result does not in general hold for an arbitrary hypergraph. In this paper, we will provide a generalization of Hanlon's result and study instances where the absolute value of the coefficient of $\lambda^{j}$ in the chromatic polynomial of $H$ is equal to the dimension of $H_{*}^{(j)}(\Delta(H))$.

\section{The Relationship Between the Chromatic Polynomial of $H$ and $\Delta(H)$}

In this section, we will provide a generalization of Hanlon's result to hypergraphs. In our study, we will need the following definition:

Let $X^{(j)}$ denote the Euler Characteristic of the $j^{\text {th }}$ Hodge piece of $\Delta(H)$. In particular,

$$
\begin{aligned}
X^{(j)} & =\sum_{i=-1}^{n-r-1}(-1)^{i} \operatorname{dim}\left(C_{i}^{(j)}(\Delta(H))\right. \\
& =\sum_{i=-1}^{n-r-1}(-1)^{i} \operatorname{dim}\left(H_{i}^{(j)}(\Delta(H))\right.
\end{aligned}
$$

Theorem 4.1 Let $H$ be a hypergraph on $n$ vertices. Then

$$
X^{(j)}(\Delta(H))=-\left[\lambda^{j}\right]\left(\chi_{H}(-\lambda)-(-\lambda)^{n}\right) .
$$

where $\left[\lambda^{j}\right] \chi_{H}(-\lambda)$ denotes the coefficient of $\lambda^{j}$ in $\chi_{H}(-\lambda)$.

Hultman [7] notes that a hypergraph $H$ on $n$ vertices, without inclusions among edges, may be associated with a subspace arrangement embeddable in the braid arrangement $A_{n}$. Following his construction and Theorem 5.7 of his paper, he notes that this then gives an interpretation of the chromatic polynomial of such hypergraphs in terms of Hilbert polynomials. Thus, for such hypergraphs, Theorem 4.1 above gives a relationship between the Euler Characteristics of the Hodge pieces of $\Delta(H)$ and coefficients in Hilbert polynomials.

\section{Star Hypergraphs}

The previous theorem leads to the following

Question 1 For which hypergraphs $H$ is it the case that, for each $j$, there exists at most one $r$ for which $\operatorname{dim}\left(H_{r}^{(j)}(\Delta(H))\right.$ is nonzero and therefore

$$
\operatorname{dim}\left(H_{r}^{(j)}(\Delta(H))=(-1)^{r+1}\left[\lambda^{j}\right]\left(\chi_{H}(-\lambda)-(-\lambda)^{n}\right) ?\right.
$$

Recall the following definition:

Definition 5.1 A simplicial complex $\Delta$ is Cohen-Macaulay over a ring $R$ if $H_{i}\left(\operatorname{link}_{\Delta}(\sigma) ; R\right)=0$ for all $\sigma \in \Delta$ and $i<\operatorname{dim}\left(\operatorname{link}_{\Delta}(\sigma)\right)$. 
Notice that if $\Delta(H)$ is Cohen-Macaulay, then the above statement is true. In this section, we will show that if $H$ is a star hypergraph, then $\Delta(H)$ is Cohen-Macaulay and thus satisfies the condition in Question 1 .

Definition 5.2 Let $H$ be a uniform hypergraph of rank $k$ with no singleton vertices. $H$ is a star hypergraph if all of its hyperedges intersect in a common set of size $k-1$.

Before we show that $\Delta(H)$ is Cohen-Macaulay, we need the following definitions.

Definition 5.3 A simplicial complex is pure if all of its maximal faces have the same dimension.

The definition of a constructible complex is due to Hochster [6].

Definition 5.4 1. Simplices, including the empty set, are constructible.

2. If $\Delta_{1}$ and $\Delta_{2}$ are $n$-dimensional constructible complexes and $\Delta_{1} \cap \Delta_{2}$ is an $(n-1)$-dimensional constructible complex, then $\Delta_{1} \cup \Delta_{2}$ is constructible.

As noted in Björner [1], constructible complexes are Cohen-Macaulay. We will show that for $H$, a star hypergraph, $\Delta(H)$ is constructible and thus Cohen-Macaulay.

Theorem 5.5 If $H$ is a star hypergraph, then $\Delta(H)$ is constructible, and hence,

$$
\begin{aligned}
\operatorname{dim}\left(H_{n-k-1}^{(j)}(\Delta(H))\right) & =(-1)^{n-k}\left[\lambda^{j}\right]\left(\chi_{H}(-\lambda)-(-\lambda)^{n}\right) . \\
& =(-1)^{n-k}\left[\lambda^{j}\right]\left(-\lambda(-\lambda-1)^{n-(k-1)}-(-\lambda)^{n-(k-1)}\right)
\end{aligned}
$$

Corollary 5.6 If $H$ is a star hypergraph, then the dimension of $H_{n-k-1}(\Delta(H))$ equals the absolute value of the sum of the coefficients of $\chi_{H}(\lambda)$ minus one.

\section{Cohen-Macaulay Hodge Subcomplexes of $\Delta(H)$}

Under certain conditions, while $\Delta(H)$ may not be Cohen-Macaulay, the Hodge subcomplexes of $\Delta(H)$ are Cohen-Macaulay and thus provide an alternative answer to Question 1. In this section, we will study these conditions. We begin by examining a class of hypergraphs which are Cohen-Macaulay when $n \leq 5$.

Theorem 6.1 Let $H$ be a uniform hypergraph of rank $k \neq 2$ having $n$ vertices, $n \leq 5$. If each hyperedge of $H$ intersects at least one other hyperedge of $H$ in a set of size $k-1$, then $\Delta(H)$ is Cohen-Macaulay and hence,

$$
\operatorname{dim}\left(H_{n-k-1}^{(j)}(\Delta(H))\right)=(-1)^{n-k}\left[\lambda^{j}\right]\left(\chi_{H}(-\lambda)-(-\lambda)^{n}\right) .
$$

We now present some results concerning the homology of hypergraphs built from $H$ by including an additional edge, beginning with two lemmas concerning their homology. For any hypergraph $H$, and any hyperedge $e$ of $H$, it can be seen directly from the definitions that there exists a short exact sequence

$$
0 \rightarrow C_{r}(\Delta(E)) \rightarrow C_{r}(\Delta(H)) \rightarrow C_{r}(\Delta(H)) / C_{r}(\Delta(E)) \rightarrow 0
$$

where $\Delta(E)$ denotes the edge complex of $e$ and $C_{*}(\Delta(H), \Delta(E))$ in degree $r$ is understood to represent the vector space spanned by ordered partitions in $C_{r}(\Delta(H))$ with no block containing $e$; the boundary maps on $C_{*}(\Delta(H), \Delta(E))$ are induced by the boundary maps on $C_{*}(\Delta(H))$. We can show 
Lemma 6.2 Let $H$ be a uniform hypergraph of rank $k$ and e be a hyperedge of $H$. Then, for $r<n-k-1$, $H_{r}(\Delta(H)) \cong H_{r}(\Delta(H), \Delta(E))$ and $H_{n-k-1}(\Delta(H)) \cong H_{n-k-1}(\Delta(E)) \oplus H_{n-k-1}(\Delta(H), \Delta(E))$.

Lemma 6.3 Consider a uniform, rank $k$ hypergraph $H$ on $n$ vertices such that $H-e$ is constructible for some hyperedge e in the edge set of $H$. Then, for $r<n-k-1, H_{r}(\Delta(H)) \cong H_{r-1}(\Delta(H / e))$.

From this lemma we can deduce:

Corollary 6.4 Let $H$ be a uniform, rank $k$ hypergraph on $n$ vertices such that, for some hyperedge e, $H-e$ is a star hypergraph and the largest size of any intersection of e with another edge of $H$ is 1 . Then the homology of $\Delta(H)$ is nonzero in dimensions $n-k-1$ and $n-2 k+1$. The dimension of $H_{n-k-1}(\Delta(H))$ equals the sum of the absolute values of the coefficients of $\chi_{H-e}(\lambda)$, and the dimension of $H_{n-2 k+1}(\Delta(H))$ equals the sum of the absolute values of the coefficients of $\chi_{H / e}(\lambda)$ minus one.

It is more difficult to analyze the case where $H$ is a uniform, rank $k$ hypergraph on $n$ vertices such that, for some hyperedge $e, H-e$ is a star hypergraph and the largest size of any intersection of $e$ with another edge of $H$ is greater than one. It would be interesting, however, to know whether there is a nice formula for the dimensions of $H_{r}(\Delta(H))$ in this case.

We now have the following theorem from which we can deduce an answer to Question 1.

Theorem 6.5 Let $H$ be a uniform hypergraph of rank $k \neq 2$ having $n$ vertices, $n \leq 5$, then all Hodge subcomplexes of $\Delta(H)$ are Cohen-Macaulay.

Notice that this result is no longer true when $n=6$ :

Example 6.6 Let $H$ be the hypergraph with 6 vertices and edges $\{1234\},\{1256\}$, and $\{3456\}$. It is straightforward to see that the dimensions of the Hodge pieces of the homology of $\Delta(H)$ are:

$$
\begin{array}{lll}
\operatorname{dim}\left(H_{1}^{(1)}(\Delta(H))\right)=0 & \operatorname{dim}\left(H_{0}^{(1)}(\Delta(H))\right)=2 & \operatorname{dim}\left(H_{-1}^{(1)}(\Delta(H))\right)=0 \\
\operatorname{dim}\left(H_{1}^{(2)}(\Delta(H))\right)=3 & \operatorname{dim}\left(H_{0}^{(2)}(\Delta(H))\right)=3 & \\
\operatorname{dim}\left(H_{1}^{(3)}(\Delta(H))\right)=3 & &
\end{array}
$$

These results lead to the following open questions:

Question 2 Is there a general condition for uniform hypergraphs with $n \geq 6$ vertices so that the Hodge subcomplexes of $\Delta(H)$ are Cohen-Macaulay?

Question 3 Let $H$ be a uniform hypergraph of rank $k$. Is there a general formula for the dimensions of the homology groups of $\Delta(H)$ ?

\section{The Chromatic Polynomial of $\Delta(H)$ and the Hodge Decompo- sition of $H_{*}(\Delta(H))$}

When each of the Hodge subcomplexes of $\Delta(H)$ have exactly one nonzero Hodge piece, there are some interesting relationships between the signs of the coefficients of the chromatic polynomial of $\Delta(H)$ and the Hodge decompositions of the homology groups of $\Delta(H)$. We present these results below.

Theorem 7.1 Suppose the $j^{\text {th }}$ Hodge subcomplex of $\Delta(H)$ has at most one nonzero Hodge piece for all j. If both 
1. the coefficient of $\lambda^{j}$ has the same sign as the coefficient of $\lambda^{j-1}$ and

2. the homology of the $j^{\text {th }}$ Hodge subcomplex is nonzero in the decomposition of $H_{k}(\Delta(H))$

then the $(j-1)^{\text {st }}$ Hodge subcomplex is nonzero in the decomposition of $H_{l}(\Delta(H))$, where l is the largest integer, with the opposite parity as $k$, that is less than $k$ and greater than -1 , and for which $H_{l}(\Delta(H))$ is nonzero.

Consider the following example:

Example 7.2 Let $H$ be the hypergraph of 5 vertices with edges $\{1,2,3\},\{2,4,5\}$, and $\{3,4,5\}$. It can be verified that $\chi_{H}(\lambda)=\lambda^{5}-3 \lambda^{3}+\lambda^{2}+\lambda$, and the dimensions of the Hodge pieces of the homology of $\Delta(H)$ are:

$$
\begin{array}{llll}
\operatorname{dim}\left(H_{1}^{(1)}(\Delta(H))\right)=0 & \operatorname{dim}\left(H_{0}^{(1)}(\Delta(H))\right)=1 & \operatorname{dim}\left(H_{-1}^{(1)}(\Delta(H))\right)=0 \\
\operatorname{dim}\left(H_{1}^{(2)}(\Delta(H))\right)=1 & \operatorname{dim}\left(H_{0}^{(2)}(\Delta(H))\right)=0 & \\
\operatorname{dim}\left(H_{1}^{(3)}(\Delta(H))\right)=3 & &
\end{array}
$$

In this case, the coefficient of $\lambda^{2}$ and $\lambda$ have the same parity, and $\operatorname{dim}\left(H_{1}^{(2)}(\Delta(H))\right)=1$. Notice that 0 is the only integer less than 1 and greater than -1 which is even. Thus, according to the theorem, $\operatorname{dim}\left(H_{0}^{(1)}(\Delta(H))=1\right.$.

Similarly, one can show that:

Theorem 7.3 Suppose the $j^{\text {th }}$ Hodge subcomplex of $\Delta(H)$ has at most one nonzero Hodge piece for all j. If both

1. the coefficient of $\lambda^{j}$ has the opposite sign as the coefficient of $\lambda^{l}$ where $l$ is the largest integer less than $j$ such that $l$ has the same parity as $j$ (and all coefficients of $\lambda^{i}$ are zero for $l<i<j$ ) and

2. if the homology of the $j^{\text {th }}$ Hodge subcomplex is nonzero in the decomposition of $H_{k}(\Delta(H))$

then the $l^{\text {th }}$ Hodge subcomplex is nonzero in the decomposition of $H_{m}(\Delta(H))$, where $m$ is the largest integer, with the opposite parity as $l$, that is less than $k$ and greater than -1 , and for which $H_{m}(\Delta(H))$ is nonzero.

Example 7.4 Let $H$ be the hypergraph of 5 vertices with edges $\{1,2,3\}$ and $\{3,4,5\}$. As determined earlier in the paper, $\chi_{H}(\lambda)=\lambda^{5}-2 \lambda^{3}+\lambda$, and the dimensions of the Hodge pieces of the homology of $\Delta(H)$ are:

$$
\begin{array}{llll}
\operatorname{dim}\left(H_{1}^{(1)}(\Delta(H))\right)=0 & \operatorname{dim}\left(H_{0}^{(1)}(\Delta(H))\right)=1 & \operatorname{dim}\left(H_{-1}^{(1)}(\Delta(H))\right)=0 \\
\operatorname{dim}\left(H_{1}^{(2)}(\Delta(H))\right)=0 & \operatorname{dim}\left(H_{0}^{(2)}(\Delta(H))\right)=0 \\
\operatorname{dim}\left(H_{1}^{(3)}(\Delta(H))\right)=2 & &
\end{array}
$$

\section{Acknowledgement}

The authors thank Philip Hanlon for his suggestion of the problem. 


\section{References}

[1] A. Björner, "Topological methods", in Handbook of Combinatorics, R. Graham, M. Grötschel, and L. Lovász (Eds.), North-Holland/Elsevier, Amsterdam, 1995, pp. 1819-1872.

[2] M. Gerstenhaber and S. D. Schack, "The shuffle bialgebra and the cohomology of commutative algebras", J. Pure Appl. Algebra, 70, No. 3 (1991), 263-272.

[3] P. Hanlon, "A Hodge decomposition interpretation for the coefficients of the chromatic polynomial", Proc. Amer. Math. Soc., 136 (2008), 3741-3749.

[4] P. Hanlon, "Hodge structures on posets," Proc. Amer. Math. Soc., 134 (2006), 1857-1867.

[5] P. Hanlon, "The action of $S_{n}$ on the components of the Hodge decomposition of Hochschild homology”, Michigan Math. J., 37 (1990), 105-124.

[6] M. Hochster, "Rings of invariants of tori, Cohen-Macaulay rings generated by monomials, and polytopes," Ann. Math., 96(1972), 318-337.

[7] A. Hultman, "Link complexes of subspace arrangements," European J. Combinatorics, 28 (2007), $781-790$.

[8] J. Jonsson, “The topology of the coloring complex”, J. Alg. Combinatorics, 21(2005), 311-329.

[9] J.-L. Loday, "Partition eulerienne et operations en homologie cyclique", C. R. Acad. Sci. Paris Ser. I Math., 307 (1988), 283-286.

[10] E. Steingrímsson, "The coloring ideal and coloring complex of a graph,” J. Alg. Comb. 14 (2001), 73-84.

[11] G. Whittle, “A geometric theory of hypergraph coloring”, Aequationes Math., 43 (1992), 45 - 58. 